\title{
The Freedom of Yetter-Drinfeld Hopf Algebras
}

\author{
Yanhua Wang \\ School of Mathematics, Shanghai University of Finance and Economics, Shanghai, China \\ Email: yhw@mail.shufe.edu.cn
}

Received 1 August 2014; revised 2 September 2014; accepted 13 September 2014

Copyright (C) 2014 by author and Scientific Research Publishing Inc.

This work is licensed under the Creative Commons Attribution International License (CC BY). http://creativecommons.org/licenses/by/4.0/

c. (i) Open Access

\section{Abstract}

In this paper, the fundamental theorem of Yetter-Drinfeld Hopf module is proved. As applications, the freedom of tensor and twisted tensor of two Yetter-Drinfeld Hopf algebras is given. Let $A$ be a Yetter-Drinfeld Hopf algebra. It is proved that the category of $A$-bimodule is equivalent to the category of $A \otimes A$-twisted module.

\section{Keywords}

\section{Hopf Algebra, Hopf Module, Yetter-Drinfeld Module, Yetter-Drinfeld Hopf Algebra}

\section{Introduction}

Let $k$ be a field and $A$ an algebra. A left $A$-module is a $k$-vector space $V$ together with a $k$-linear map $A \otimes V \rightarrow V$ such that $a b \rightarrow v=a \rightarrow(b \rightarrow v)$ and $1 \rightarrow v=v$. The category of left $A$-module is denoted by ${ }_{A} M$. Dually, let $(C, \Delta, \varepsilon)$ be a coalgebra. A left $C$-comodule is a $k$-vector space $V$ together with a $k$ -linear map $\rho: V \rightarrow V \otimes C$ such that

$$
\sum\left(a^{-1}\right)_{1} \otimes\left(a^{-1}\right)_{2} \otimes a^{0}=\sum a^{-1} \otimes\left(a^{0}\right)^{-1} \otimes\left(a^{0}\right)^{0}, \quad \sum \varepsilon\left(a^{-1}\right) a^{0}=a .
$$

The category of left $C$-comodule is denoted by ${ }^{C} M$. For more about modules and comodules, see [1]-[3].

Assume that $H$ is a Hopf algebra with antipode $S$, a left Yetter-Drinfeld module over $H$ is a $k$-vector space $V$ which is both a left $H$-module and left $H$-comodule and satisfies the compatibility condition

$$
\sum(h \rightarrow v)^{-1} \otimes(h \rightarrow v)^{0}=\sum h_{1} v^{-1} S\left(h_{3}\right) \otimes h_{2} \rightarrow v^{0}
$$

for all $h \in H, v \in V$. The category of left Yetter-Drinfeld module is denoted by ${ }_{H}^{H} Y D$. Yetter-Drinfeld modules category constitutes a monomidal category, see [4]. The category is pre-braided; the pre-braiding is given by 


$$
\tau_{V, W}: V \otimes W \rightarrow W \otimes V, \quad v \otimes w \mapsto\left(v^{-1} \rightarrow w\right) \otimes v^{0} .
$$

The map is a braiding in ${ }_{H}^{H} Y D$ precisely when Hopf algebra $H$ has a bijective antipode $S$ with inverse $\bar{S}$ of $S$. In this case, the inverse of $\tau_{V, W}$ is

$$
\tau_{V, W}^{-1}: W \otimes V \rightarrow V \otimes W, \quad w \otimes v \mapsto \sum v^{0} \otimes \bar{S}\left(v^{-1}\right) \rightarrow w .
$$

Let $H$ be a Hopf algebra and ${ }_{H}^{H} Y D$ the category of left Yetter-Drinfeld module over $H$. We call $A$ a Hopf algebra in ${ }_{H}^{H} Y D$ or Yetter-Drinfeld Hopf algebra if $A$ is a $k$-algebra and a $k$-coalgebra, and the following conditions (a1)-(a6) hold for $h \in H, a, b \in A$,

(a1) $A$ is a left $H$-module algebra, i.e.,

$$
h \rightarrow(a b)=\sum\left(h_{1} \rightarrow a\right)\left(h_{2} \rightarrow b\right), \quad h \rightarrow 1_{A}=\varepsilon(h) 1_{A} .
$$

(a2) $A$ is a left $H$-comodule algebra, i.e.,

$$
\rho(a b)=\sum(a b)^{-1} \otimes(a b)^{0}=\sum a^{-1} b^{-1} \otimes a^{0} b^{0}, \quad \rho\left(1_{A}\right)=1_{H} \otimes 1_{A} .
$$

(a3) $A$ is a left $H$-module coalgebra, i.e.,

$$
\Delta(h \rightarrow a)=\sum\left(h_{1} \rightarrow a_{1}\right) \otimes\left(h_{2} \rightarrow a_{2}\right), \quad \varepsilon(h \rightarrow a)=\varepsilon_{H}(h) \varepsilon_{A}(a) .
$$

(a4) $A$ is a left $H$-comodule coalgebra, i.e.,

$$
\sum a^{-1} \otimes\left(a^{0}\right)_{1} \otimes\left(a^{0}\right)_{2}=\sum a_{1}^{-1} a_{2}^{-1} \otimes a_{1}^{0} \otimes a_{2}^{0}, \quad \sum a^{-1} \varepsilon_{A}\left(a^{0}\right)=\varepsilon_{A}(a) 1_{H} .
$$

(a5) $\Delta, \varepsilon$ are algebra maps in ${ }_{H}^{H} Y D$, i.e.,

$$
\Delta(a b)=\sum a_{1}\left(a_{2}^{-1} \rightarrow b_{1}\right) \otimes a_{2}^{0} b_{2}, \quad \Delta(1)=1 \otimes 1, \quad \varepsilon(a b)=\varepsilon(a) \varepsilon(b), \quad \varepsilon\left(1_{A}\right)=1_{k} .
$$

(a6) There exists a $k$-linear map $S: A \rightarrow A$ in ${ }_{H}^{H} Y D$ such that

$$
\sum S\left(a_{1}\right) a_{2}=\varepsilon(a) 1_{A}=\sum a_{1} S\left(a_{2}\right)
$$

One easily get that $S$ is both $H$-linear and $H$-colinear. In general, Yetter-Drinfeld Hopf algebras are not ordinary Hopf algebras because the bialgebra axiom asserts that they obey (a5). However, it may happen that Yetter-Drinfeld Hopf algebras are ordinary Hopf algebras when the pre-braiding is trivial, for details see [5].

Yetter-Drinfeld Hopf algebras are generalizations of Hopf algebras. Some important properties of Hopf algebras can be applied to Yetter-Drinfeld Hopf algebra. For example: Doi gave the trace formular of Yetter-Drinfeld Hopf algebras in [6] and studied Hopf module in [7]; Chen and Zhang constructed Four-dimensional Yetter-Drinfeld module algebras in [8]; Zhu and Chen studied Yetter-Drinfeld modules over the Hopf-Ore Extension of Group algebra of Dihedral group in [9]; Alonso Álvarez, Fernández Vilaboa, González Rodríguez and Soneira Calvoar considered Yetter-Drinfeld modules over a weak braided Hopf algebra in [10], and so on.

Hopf module fundamental theorem plays an important role in Hopf algebras. This theory can be generalized to Yetter-Drinfel Hopf algebras.

Theorem 1.1. Let $A$ be a Yetter-Drinfeld Hopf algebra, $M \in_{A}^{A} M$ be a Yetter-Drinfeld Hopf module, then $M \cong A \otimes{ }^{c o A} M$ as left $A$ Yetter-Drinfeld Hopf module.

Note that Theorem 1.1 was appeared in [7], but we give a different proof with Doi's here.

Let $A$ be a Yetter-Drinfeld Hopf algebra. Define the multiplication of $(A \otimes A)^{\tau}$ as

$$
(a \otimes b)(c \otimes d)=\sum a\left(b^{-1} \rightarrow c\right) \otimes b^{0} d,
$$

then $(A \otimes A)^{\tau}$ is an algebra. But it is not a Yetter-Drinfeld Hopf algebra if $\tau$ is not the trivial twist T. As applications of Yetter-Drinfeld Hopf module fundamental theory, we have the freedom of the tensor of Yetter-Drinfeld Hopf algebras and twisted tensor of Yetter-Drinfeld Hopf algebras.

Theorem 1.2. Let $A$ be a Yetter-Drinfeld Hopf algebra, then $A \otimes A$ and $(A \otimes A)^{\tau}$ are free over $A$. 
We also proved the category of Yetter-Drinfeld. A -bimodule is equivalent to the category of $(A \otimes A)^{\tau}$ module.

Theorem 1.3. Let $A$ be a Yetter-Drinfeld Hopf algebra. Then the category of ${ }_{A} M_{A}$ and ${ }_{(A \otimes A)^{\tau}} M$ are equivalent.

\section{The Freedom of Yetter-Drinfeld Hopf Algebras}

In this section, we require $H$ is a Hopf algebra and $A$ is a Yetter-Drinfeld module over $H$. Moreover, we need $A$ is a Yetter-Drinfeld Hopf algebra. Next, we will give the definition of Yetter-Drinfeld Hopf module, also see [7].

Definition 2.1. Let $A$ be a Yetter-Drinfeld Hopf algebra. The Yetter-Drinfeld Hopf module over $A$ is defined by the following

1) $M$ is a left $A$-module and left $A$-comodule with comodule map $\rho_{M}: M \rightarrow A \otimes M$,

2) $\rho_{M}$ is a $A$-module map, i.e., $\rho_{M}(a m)=\sum a_{1}\left(a_{2}^{-1} \rightarrow m_{-1}\right) \otimes a_{2}^{2} m_{0}$, where $a_{1}, a_{2} \in A$.

Note that $A$ is a left $H$-comodule with $\rho_{A}(a)=\sum a^{-1} \otimes a^{0}, a \in A$, and $M$ is a left $A$-comodule with $\rho_{M}(m)=\sum m_{-1} \otimes m_{0}, m \in M$. The Yetter-Drinfeld Hopf module category over $A$ is denoted by ${ }_{A}^{A} M$.

Define ${ }^{\mathrm{COA}} M=\left\{m \mid \rho_{M}(m)=1 \otimes m\right\}$ is the set of coinvariant elelments of $M$. Next conclusion is similar to the fundamental theorem of Hopf algebra, we call it as the fundamental theorem of Yetter-Drinfeld Hopf module.

Theorem 2.2. Let $A$ be a Yetter-Drinfeld Hopf algebra, $M \in_{A}^{A} M$ be a Yetter-Drinfeld Hopf module. Then $M \cong A \otimes^{\mathrm{COA}} M$ as left $A$ Yetter-Drinfeld Hopf module.

Proof: We define $\alpha: A \otimes^{c o A} M \rightarrow M$ by $a \otimes m \mapsto a \cdot m$ and $\beta: M \rightarrow A \otimes^{c o A} M$ by

$$
m \mapsto \sum m^{-2} \otimes S\left(m^{-1}\right) \cdot m^{0} .
$$

First, we show that $\beta$ is well-defined, i.e., $\sum S\left(m_{-1}\right) \cdot m_{0} \in^{c o A} M$. In fact, we have

$$
\begin{aligned}
\rho\left(\sum S\left(m_{-1}\right) \cdot m_{0}\right) & =\sum\left(S\left(m_{-2}\right)\right)_{1}\left(S\left(m_{-2}\right)_{2}^{-1} \rightarrow m_{-1}\right) \otimes\left(S\left(m_{-2}\right)\right)_{2}^{0} \cdot m_{0} \\
& =\sum m_{-3}^{-2} \rightarrow S\left(m_{-2}\right)\left(m_{-3}^{-1} \rightarrow m_{-1}\right) \otimes S\left(m_{-3}\right)^{0} \cdot m_{0} \\
& =\sum m_{-3} \rightarrow\left(S\left(m_{-2}\right)\left(m_{-1}\right)\right) \otimes S\left(m_{-3}\right)^{0} \cdot m_{0} \\
& =\sum m_{-2} \rightarrow \varepsilon\left(m_{-1}\right) \otimes S\left(m_{-2}\right)^{0} \cdot m_{0} \\
& =\sum S\left(m_{-1}\right) \cdot m_{0} .
\end{aligned}
$$

So $\sum S\left(m_{-1}\right) m_{0} \in^{C O A} M$. Thus $\beta$ is well-defined.

We will show that $\alpha$ is the inverse of $\beta$. Indeed, if $m \in M$ we have

$$
\alpha \beta(m)=\alpha\left(\sum m_{-2} \otimes S\left(m_{-1}\right) \cdot m_{0}\right)=\sum m_{-2} S\left(m_{-1}\right) \cdot m_{0}=\sum \varepsilon\left(m_{-1}\right) m_{0}=m,
$$

Hence $\alpha \beta=i d$. Conversely, if $m \in^{c o A} M, a \in A$, then

$$
\begin{aligned}
\beta \alpha(a \otimes m)=\beta(a \cdot m) & =\sum(a \cdot m)_{-2} \otimes S(a \cdot m)_{-1} \cdot(a \cdot m)_{0} \\
& =\sum\left(a_{1}\left(a_{2}^{-1} \rightarrow m_{-1}\right)\right)_{1} \otimes S\left(\left(a_{1}\left(a_{2}^{-1} \rightarrow m_{-1}\right)\right)_{2}\right) a_{2}^{2} \cdot m_{0} \\
& =\sum a_{1} \otimes S\left(a_{2}\right) a_{3} \cdot m=a \otimes m,
\end{aligned}
$$

which show that $\beta \alpha=i d$ too. It remains to show that $\alpha$ is a morphism of $H$-module and $H$-comodule. The first assertion is clear, since

$$
\alpha(b \cdot(a \otimes m))=\alpha(b a \otimes m)=b a \cdot m=b \cdot \alpha(a \otimes m) .
$$

Next, we show that $\alpha$ is a $H$-comodule morphism, i.e. $\rho \alpha=($ id $\otimes \alpha)(\Delta \otimes i d)$. Indeed, we have 


$$
\rho \alpha(a \otimes m)=\rho(a \cdot m)=\sum a_{1}\left(a_{2}^{-1} \rightarrow m_{-1}\right) \otimes a_{2}^{0} \cdot m_{0}=\sum a_{1} \otimes a_{2} \cdot m=(i d \otimes \alpha)(\Delta \otimes i d)(a \otimes m) .
$$

This complete the proof.

Proposition 2.3. We have $A \otimes A$ is a Yetter-Drinfeld Hopf module over $A$.

Proof: $A \otimes A$ is an $A$-module by the trivial module action: $a \cdot(b \otimes c)=a b \otimes c$. In fact, for $a, b \in A$, $c \otimes d \in A \otimes A$, we have $a(b(c \otimes d))=a(b c \otimes d)=a b c \otimes d=(a b)(c \otimes d)$ and $1(c \otimes d)=c \otimes d$. The A-comodule structure of $A \otimes A$ is defined by $\rho(a \otimes b)=\sum a_{1} \otimes a_{2} \otimes b \in A \otimes A \otimes A$. It is easy to check $A \otimes A$ is an Yetter-Drinfeld Hopf module over $A$, we omit it.

Theorem 2.4. Let $A$ be a Yetter-Drinfeld Hopf algebra, then $A \otimes A$ is free over $A$.

Proof: Apply $M=A \otimes A$ to the fundamental theorem of Yetter-Drinfeld Hopf algebra, then $\alpha$ and $\beta$ become $\alpha: a \otimes(b \otimes c) \mapsto a(b \otimes c)=a b \otimes c$ and

$$
\beta: b \otimes c \mapsto \sum b_{1} \otimes S\left(b_{2}\right)\left(b_{3} \otimes c\right)=\sum b_{1} \otimes\left(S\left(b_{2}\right) b_{3} \otimes c\right)=b \otimes(1 \otimes c) .
$$

Next, we show that $\beta \alpha=i d=\alpha \beta$. In fact, we have

$$
\begin{aligned}
\beta \alpha(a \otimes(b \otimes c)) & =\beta(a b \otimes c)=\sum(a b)_{1} \otimes S\left((a b)_{2}\right) \cdot\left((a b)_{3} \otimes c\right) \\
& =\sum a_{1}\left(a_{2}^{-1} \rightarrow b_{1}\right) \otimes S\left(\left(a_{2}^{0} b_{2}\right)_{1}\right) \cdot\left(\left(a_{2}^{0} b_{2}\right)_{2} \otimes c\right) \\
& \left.=\sum a_{1}\left(a_{2}^{-1} \rightarrow b_{1}\right) \otimes S\left(\left(a_{2}^{0}\right)_{1}\left(\left(a_{2}^{0}\right)_{2}^{-1} \rightarrow b_{2}\right)\right) \cdot\left(a_{2}^{0}\right)_{2}^{0} b_{3} \otimes c\right) \\
& =\sum a_{1}\left(a_{2}^{-1} a_{3}^{-2} \rightarrow b_{1}\right) \otimes S\left(a_{2}^{0}\left(a_{3}^{-1} \rightarrow b_{2}\right)\right) \cdot\left(\left(a_{3}^{0} b_{3}\right) \otimes c\right) \\
& =\sum a_{1}\left(a_{2}^{-1} a_{3}^{-2} \rightarrow b_{1}\right) \otimes S\left(a_{3}^{-1} \rightarrow b_{2}\right) S\left(a_{2}^{0}\right) \cdot\left(\left(a_{3}^{0} b_{3}\right) \otimes c\right) \\
& =\sum a_{1}\left(a_{2}^{-1} a_{3}^{-2} \rightarrow 1\right) \otimes S\left(a_{3}^{-1} \rightarrow 1\right) S\left(a_{2}^{0}\right)\left(a_{3}^{0} b_{3} \otimes c\right) \\
& =\sum a_{1} \otimes S a_{2} \cdot\left(a_{3} b_{3} \otimes c\right) \\
& =\sum a_{1} \otimes S\left(a_{2}\right) a_{3} b_{3} \otimes c \\
& =a \otimes(b \otimes c) .
\end{aligned}
$$

and

$$
\alpha \beta(b \otimes c)=\alpha\left(\sum b_{1} \otimes S\left(b_{2}\right)\left(b_{3} \otimes c\right)\right)=\sum b_{1}\left(S\left(b_{2}\right)\left(b_{3} \otimes c\right)\right)=b \otimes c .
$$

Hence, we have $\beta \alpha=i d=\alpha \beta$.

Moreover, $\alpha$ is an $A$-module map. Since $\alpha(a(b \otimes(c \otimes d)))=\alpha(a b \otimes(c \otimes d))=a b c \otimes d \quad$ and $a \alpha(b \otimes(c \otimes d))=a(b c \otimes d)=a b c \otimes d$. Furthermore, $\alpha$ is also an $A$-comodule map by the following. Take $a \in A, b \otimes c \epsilon^{c o A}(A \otimes A)$, then $\rho(b \otimes c)=\sum b_{1} \otimes b_{2} \otimes c=1 \otimes b \otimes c$. We have

$$
\rho(\alpha(a \otimes(b \otimes c)))=\rho(a b \otimes c)=\sum(a b)_{1} \otimes(a b)_{2} \otimes c=\sum a_{1}\left(a_{2}^{-1} \rightarrow b_{1}\right) \otimes a_{2}^{0} b_{2} \otimes c=\sum a_{1} \otimes a_{2} b \otimes c
$$

and

$$
(i d \otimes \alpha)(\Delta \otimes i d)(a \otimes(b \otimes c))=(i d \otimes \alpha)\left(\sum a_{1} \otimes a_{2} \otimes b \otimes c\right)=\sum a_{1} \otimes a_{2} b \otimes c .
$$

In a word, $A \otimes A \cong A \otimes \otimes^{c o A}(A \otimes A)$, so $A \otimes A$ is free over $A$. This completes the proof.

\section{Twist Yetter-Drinfeld Hopf Module}

Let $A$ be a Yetter-Drinfeld Hopf algebra over Hopf $H$. Define the multiplication of $(A \otimes A)^{\tau}$ as follows:

$$
(a \otimes b)(c \otimes d)=\sum a\left(b^{-1} \rightarrow c\right) \otimes b^{0} d .
$$


Lemma 3.1. Let $A$ be a Yetter-Drinfeld Hopf algebra, then $(A \otimes A)^{\tau}$ is an algebra with multiplication (1).

Proof: We only need to check the associativity of $(A \otimes A)^{\tau}$

$$
\begin{aligned}
((a \otimes b)(c \otimes d))(e \otimes f) & =\sum\left(a\left(b^{-1} \rightarrow c\right) \otimes b^{0} d\right)(e \otimes f)=\sum a\left(b^{-2} \rightarrow c\right)\left(b^{-1} d^{-1} \rightarrow e\right) \otimes b^{0} d^{0} f \\
& =a\left(b^{-1} \rightarrow\left(c\left(d^{-1} \rightarrow e\right)\right)\right) \otimes b^{0} d f \\
& =(a \otimes b)\left(c\left(d^{-1} \rightarrow e\right) \otimes d f\right)=(a \otimes b)((c \otimes d)(e \otimes f)) .
\end{aligned}
$$

And $1 \otimes 1$ is the unit element of $(A \otimes A)^{\tau}$. Thus $(A \otimes A)^{\tau}$ is an algebra.

Remark 3.2. $(A \otimes A)^{\tau}$ is a Yetter-Drinfeld Hopf algebra if and only if $\tau=T$. See reference [5] for the details.

Denote the $A$-bimodule category by ${ }_{A} M_{A}$, and $(A \otimes A)^{\tau}$-module category by ${ }_{(A \otimes A)^{\tau}} M$.

Theorem 3.3. Let $A$ be a Yetter-Drinfeld Hopf algebra. Then the category of ${ }_{A} M_{A}$ and ${ }_{(A \otimes A)^{\tau}} M$ are equivalent.

Proof: we are going to construct the functor $F:_{A} M_{A} \rightarrow_{(A \otimes A)^{\tau}} M$ as follows. Let $M$ be an $A$-bimodule. We denote the two-side action on $M$ by “.”. Define $F(M)=M$ as $k$-space with the left $(A \otimes A)^{\tau}$ action given by

$$
(a \otimes b) m=\sum a \cdot\left(b^{-1} \rightarrow m\right) \cdot S\left(b_{0}\right), \quad a, b \in A, \quad m \in M .
$$

We claim that the action is well-defined, i.e. $((a \otimes b)(c \otimes d)) m=(a \otimes b)((c \otimes d) m)$. In fact, we have

$$
\begin{aligned}
((a \otimes b)(c \otimes d)) m & =\sum\left(a\left(b^{-1} \rightarrow c\right) \otimes b^{0} d\right) m=\sum \mathrm{a}\left(b^{-2} \rightarrow c\right) \cdot\left(b^{-1} d^{-1} \rightarrow m\right) \cdot S\left(b^{0} d^{0}\right) \\
& =\sum\left(a\left(b^{-3} \rightarrow c\right)\right) \cdot\left(b^{-2} d^{-1} \rightarrow m\right) \cdot\left(b^{-1} \rightarrow S\left(d^{0}\right)\right) S\left(b^{0}\right) .
\end{aligned}
$$

and

$$
\begin{aligned}
(a \otimes b)((c \otimes d) m) & =\sum(a \otimes b)\left(c \cdot\left(d^{-1} \rightarrow m\right) \cdot S\left(d^{0}\right)\right)=\sum a\left(b^{-1} \rightarrow\left(c \cdot\left(d^{-1} \rightarrow m\right) \cdot S\left(d^{0}\right)\right)\right) \cdot S\left(b^{0}\right) \\
& =\sum\left(a\left(b^{-3} \rightarrow c\right)\right) \cdot\left(b^{-2} d^{-1} \rightarrow m\right) \cdot\left(b^{-1} \rightarrow S\left(d^{0}\right)\right) S\left(b^{0}\right) .
\end{aligned}
$$

By comparing the above two identities, we have $M$ and $(A \otimes A)^{\tau}$-module.

Moreover, we have the functor $G:{ }_{(A \otimes A)^{\tau}} M \rightarrow_{A} M_{A}$ given as follows: Let $N$ be a left $(A \otimes A)^{\tau}$-module, define $G(N)$ to be $N$ as $k$-space, and its $A$-bimodule structure given by $a \cdot n=(a \otimes 1) n$ and $n \cdot b=\sum\left(1 \otimes S^{-1}\left(b^{0}\right)\right)\left(\bar{S}\left(b^{-1}\right) \rightarrow n\right)$. Note that $S^{-1}$ denotes the inverse of $S_{A}$ and $\bar{S}$ denotes the inverse of $S_{H}$. Clearly, $G(N)$ is a left $A$-module. Note that

$$
\begin{aligned}
(n \cdot a) \cdot b & =\sum\left(1 \otimes S^{-1}\left(b^{0}\right)\right)\left(\bar{S}\left(b^{-1}\right) \rightarrow(n \cdot a)\right) \\
& =\left(1 \otimes S^{-1}\left(b^{0}\right)\right)\left(\bar{S}\left(b^{-1}\right) \rightarrow\left(1 \otimes S^{-1}\left(a^{0}\right)\right)\left(\bar{S}\left(a^{-1}\right) \rightarrow n\right)\right) \\
& =\left(1 \otimes S^{-1}\left(b^{0}\right)\right)\left(1 \otimes\left(\bar{S}\left(b^{-1}\right) \rightarrow S^{-1}\left(a^{0}\right)\right)\right)\left(\bar{S}\left(b^{-2}\right) \bar{S}\left(a^{-1}\right) \rightarrow n\right) \\
& =\left(1 \otimes S^{-1}\left(b^{0}\right)\left(\bar{S}\left(b^{-1}\right) \rightarrow S^{-1}\left(a^{0}\right)\right)\right)\left(\bar{S}\left(a^{-1} b^{-2}\right) \rightarrow n\right) \\
& =\left(1 \otimes S^{-1}\left(a^{0} b^{0}\right)\right)\left(\bar{S}\left(a^{-1} b^{-1}\right) \rightarrow n\right)=n \cdot(a b) .
\end{aligned}
$$

Hence, $G(N)$ is also a right $A$-module. We have 


$$
(a \cdot n) \cdot b=(a \otimes 1) n \cdot b=\sum(a \otimes 1)\left(1 \otimes S^{-1}\left(b^{0}\right)\right)\left(\bar{S}\left(b^{-1}\right) \rightarrow n\right)
$$

and

$$
a \cdot(n \cdot b)=\sum a \cdot\left(1 \otimes S^{-1}\left(b^{0}\right) \bar{S}\left(b^{-1}\right) \rightarrow n\right)=\sum(a \otimes 1)\left(1 \otimes S^{-1}\left(b^{0}\right)\right)\left(\bar{S}\left(b^{-1}\right) \rightarrow n\right),
$$

therefore, $G(N)$ is a $A$-bimodule. It is easy to check that the functors $F$ and $G$ are inverse to each other. This completes the proof.

Let $A$ be a Yetter-Drinfeld Hopf algebra, then $(A \otimes A)^{\tau}$ is a right $A$-module by

$$
(a \otimes b) \cdot c \mapsto \sum a\left(b^{-1} \rightarrow c_{1}\right) \otimes b^{0} c_{2} .
$$

Recall that if $V$ is a vector space, then $V \otimes A$ is a free $A$-module with the action $(v \otimes a) b=v \otimes a b$.

Theorem 3.4. The right $A$-module $(A \otimes A)^{\tau}$ defined above is free over $A$.

Proof: Let $V$ denote the underlying space of $A$. Thus $V \otimes A$ become a right free module. Define a map $\psi:(A \otimes A)^{\tau} \rightarrow V \otimes A: a \otimes b=\sum a S\left(b_{1}\right) \otimes b_{2}$. It is obvious that $\psi$ is a bijection with inverse $\psi^{-1}(a \otimes b)=\sum a b_{1} \otimes b_{2}$. We claim that $\psi$ is a right $A$-module morphism, then we are done.

In fact, we have

$$
\begin{aligned}
\psi((a \otimes b) c) & =\sum \psi\left(a\left(b^{-1} \rightarrow c_{1}\right) \otimes b^{0} c_{2}\right) \\
& =\sum \mathrm{a}\left(b^{-1} \rightarrow c_{1}\right) S\left(\left(b^{0} c_{2}\right)_{1}\right) \otimes\left(b^{0} c_{2}\right)_{2} \\
& =\sum a\left(b^{-1} \rightarrow c_{1}\right) S\left(b_{1}^{0}\left(\left(b_{2}^{0}\right)^{-1} \rightarrow c_{2}\right)\right) \otimes b_{2}^{0} c_{3} \\
& =\sum a\left(b_{1}^{-1} b_{2}^{-2} \rightarrow c_{1}\right) S\left(b_{1}^{0}\left(b_{2}^{-1} \rightarrow c_{2}\right)\right) \otimes b_{2}^{0} c_{3} \\
& =\sum a\left(b_{1}^{-2} b_{2}^{-2} \rightarrow c_{1}\right)\left(b_{1}^{-1}\left(b_{2}^{-1} \rightarrow S\left(c_{2}\right)\right)\right) S\left(b_{1}^{0}\right) \otimes b_{2}^{0} c_{3} \\
& =\sum a\left(b_{1}^{-1} b_{2}^{-1} \rightarrow c_{1} S\left(c_{2}\right)\right) S\left(b_{1}^{0}\right) \otimes b_{2}^{0} c_{3} \\
& =\sum a\left(b_{1}^{-1} b_{2}^{-1} \rightarrow \varepsilon\left(c_{1}\right)\right) S\left(b_{1}^{0}\right) \otimes b_{2}^{0} c_{2} \\
& =\sum a \varepsilon\left(b_{1}^{-1}\right) \varepsilon\left(b_{2}^{-1}\right) S\left(b_{1}^{0}\right) \otimes b_{2}^{0} c \\
& =\sum a S\left(b_{1}\right) \otimes b_{2} c .
\end{aligned}
$$

Note that the right $A$-module structure on $V \otimes A$ is $(v \otimes a) \cdot b=v \otimes a b$, so $\psi(a \otimes b) \cdot c=\sum a S\left(b_{1}\right) \otimes b_{2} c$. Thus we have proved that $\psi((a \otimes b) \cdot c)=\psi(a \otimes b) \cdot c$. This completes the proof.

\section{Acknowledgements}

Supported by the National Nature Science Foundation of China (Grant No. 11271239).

\section{References}

[1] Dascalescu, S., Nastasescu, C. and Raianu, S. (2001) Hopf Algebras; An Introduction. Marcel Dekker, Inc., New York.

[2] Montgomery, S. (1993) Hopf Algebras and Their Actions on Rings. CBMS Regional Conf. Series in Math. 82, American Mathematical Society, Providence, RI.

[3] Sweedler, M.E. (1969) Hopf Algebras. Benjamin, New York.

[4] Yetter, D.N. (1990) Quantum Groups and Representation of Monoidal Categories. Mathematical Proceedings of the Cambridge Philosophical Society, 108, 261-290. http://dx.doi.org/10.1017/S0305004100069139

[5] Sommerhauser, Y. (2002) Yetter-Drinfeld Hopf Algebras over Groups of Prime Order. Vol. 1789, Springer, Berlin.

[6] Doi, Y. (2000) The Trace Formula for Braided Hopf Algebras. Communications in Algebra, 28, 1881-1895. http://dx.doi.org/10.1080/00927870008826933 
[7] Doi, Y. (1998) Hopf Module in Yetter-Drinfeld Module Categories. Communications in Algebra, 26, 3057-3070. http://dx.doi.org/10.1080/00927879808826327

[8] Chen, H.X. and Zhang, Y. (2006) Four-Dimensional Yetter-Drinfeld Module Algebras. Journal of Algebra, 296, 582634. http://dx.doi.org/10.1016/j.jalgebra.2005.08.011

[9] Zhu, H. and Chen H.X. (2012) Yetter-Drinfeld Modules over the Hopf-Ore Extension of Group Algebra of Dihedral Group. Acta Mathematica Sinca, 28, 487-502. http://www.cnki.com.cn/Article/CJFDTotal-ACMS201203007 http://dx.doi.org/10.1007/s10114-011-9777-4

[10] Alonso Álvarez, J.N., Fernández Vilaboa, J.M., González Rodríguez, R. and Soneira Calvoar, C. The Monoidal Category of Yetter-Drinfeld Modules over a Weak Braided Hopf Algebra. arXiv: 1203.2474. http://arxiv.org/abs/1203.2474 
Scientific Research Publishing (SCIRP) is one of the largest Open Access journal publishers. It is currently publishing more than 200 open access, online, peer-reviewed journals covering a wide range of academic disciplines. SCIRP serves the worldwide academic communities and contributes to the progress and application of science with its publication.

Other selected journals from SCIRP are listed as below. Submit your manuscript to us via either submit@scirp.org or Online Submission Portal.
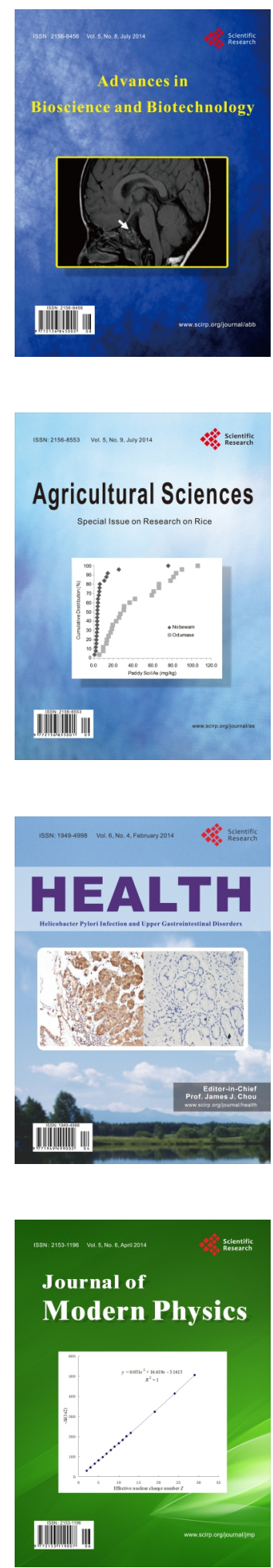
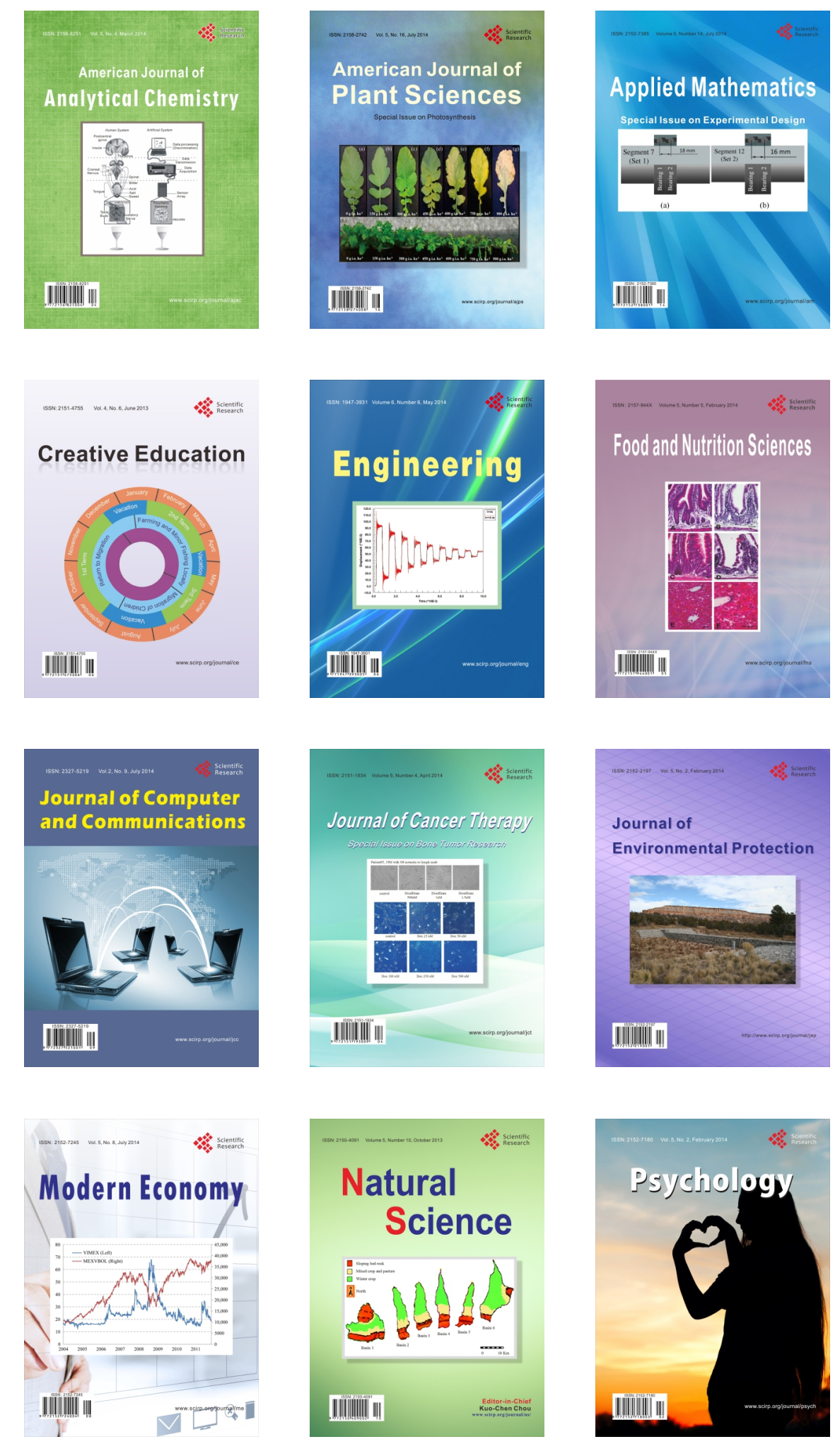